Revue d'histoire de l'Amérique française

REVUE D.HISTOIRE DE L'AMÉRIQUE FRANÇAISE

\title{
Fuir la ville
}

\section{Villégiature et villégiateurs dans la région de Montréal,} 1890-1940

\section{Michèle Dagenais}

\section{Volume 58, numéro 3, hiver 2005}

URI : https://id.erudit.org/iderudit/011624ar

DOI : https://doi.org/10.7202/011624ar

Aller au sommaire du numéro

\section{Éditeur(s)}

Institut d'histoire de l'Amérique française

ISSN

0035-2357 (imprimé)

1492-1383 (numérique)

Découvrir la revue

Citer cet article

Dagenais, M. (2005). Fuir la ville : villégiature et villégiateurs dans la région de Montréal, 1890-1940. Revue d'histoire de l'Amérique française, 58(3), 315-345. https://doi.org/10.7202/011624ar

\section{Résumé de l'article}

La présence importante de l'eau et l'abondance de rives dans la région montréalaise ont attiré des générations de citadins en quête de lieux de loisirs et de vie, proches de la nature. Cet article met au jour la manière dont évoluent les rapports des villégiateurs à leur environnement naturel entre 1890 et 1940. Pour multiples que soient leurs définitions, les villégiateurs partagent une vision instrumentale de la nature, considérée comme un moyen pour se constituer des milieux de vie sur mesure, servant à l'affirmation de leur pouvoir et de leur prestige tout comme à la consolidation de leur identité sociale. Terme dont la puissance d'évocation est sans limites, la nature ne livre pas elle-même son propre sens. Ses significations se construisent à travers les valeurs dont elle est investie, les rêves qu'elle nourrit, les projets et les usages auxquels elle donne vie.
Tous droits réservés @ Institut d'histoire de l'Amérique française, 2005
Ce document est protégé par la loi sur le droit d'auteur. L'utilisation des services d'Érudit (y compris la reproduction) est assujettie à sa politique d'utilisation que vous pouvez consulter en ligne.

https://apropos.erudit.org/fr/usagers/politique-dutilisation/ 


\title{
Fuir la ville :
}

villégiature et villégiateurs dans la région de Montréal, I89o-r940

\author{
MICHÈLE DAGENAIS \\ Département d'histoire \\ Université de Montréal
}

RÉSUMÉ • La présence importante de l'eau et l'abondance de rives dans la région montréalaise ont attiré des générations de citadins en quête de lieux de loisirs et de vie, proches de la nature. Cet article met au jour la manière dont évoluent les rapports des villégiateurs à leur environnement naturel entre 1890 et 1940. Pour multiples que soient leurs définitions, les villégiateurs partagent une vision instrumentale de la nature, considérée comme un moyen pour se constituer des milieux de vie sur mesure, servant à l'affirmation de leur pouvoir et de leur prestige tout comme à la consolidation de leur identité sociale. Terme dont la puissance d'évocation est sans limites, la nature ne livre pas elle-même son propre sens. Ses significations se construisent à travers les valeurs dont elle est investie, les rêves qu'elle nourrit, les projets et les usages auxquels elle donne vie.

ABSTRACT - The predominance of water and the abundance of riverbanks in the Montreal region have attracted generations of urban residents in search of living and leisure spaces located close to nature. This article sheds light upon the evolution of the rapport between vacationers and their natural environment between 1890 and 1940. In spite of the multiplicity of definitions, vacationers shared an instrumental vision of nature, which was seen as a

1. Les recherches à l'origine de cet article ont été réalisées grâce à des subventions d'équipe du FQRSC (2002-2005) et du CRSHC (2003-2006). Je remercie Gillian Leitch et Caroline Aubin des Roches pour leur patient et minutieux travail de dépouillement des journaux. J'ai d'abord eu l'occasion de discuter des résultats de ces recherches lors de la Urban History Group Conference, Royal Holloway College, University of London, les $1^{\text {er }}$ et 2 avril 2004, qui avait pour thème : "The Nineteenth Century Suburb and Beyond ». Je suis reconnaissante envers Dominique Deslandres et Claire Poitras pour leur aide dans l'élaboration de ma réflexion. Je remercie également Christian Laville pour sa lecture rigoureuse de l'article et ses suggestions, de même que les évaluateurs anonymes de la Revue dont les commentaires constructifs m’ont incitée à préciser certaines des questions abordées. 
means of fashioning a tailor-made living environment that served to assert both their power and their prestige and that consolidated their social identity. Given the infinite suggestive possibilities of the term, the definition of nature was in no way self-evident. Its meanings were constructed through the values invested in it, the dreams it nourished and the projects to which it gave life.

Imaginez une résidence en montagne qui répond parfaitement à vos aspirations... un endroit à l'abri de la cohue et de l'agitation, où vous pouvez vraiment déconnecter tout en restant branché sur votre monde.

Il y a longtemps que vous rêvez de fuir la ville, en continuant à profiter de ses avantages? Alors n'attendez plus. Le Cercle... vous propose un style de vie à la fois champêtre, convivial... qui répond à toutes vos attentes en termes d'élégance et de bien-être ${ }^{2}$.

Les rives... de la rivière Châteauguay sont encore, cette année, le point de rendez-vous... pour aller jouir de la tranquillité de la campagne et en respirer l'air frais... On y trouve encore l'une des rivières les plus poissonneuses se jetant un peu plus loin dans le magnifique lac Saint-Louis, ce qui, naturellement, fait les délices du pêcheur à la ligne et de l'amateur de tous les sports nautiques... Ce n'est qu'à quelques milles de Montréal... que l'on trouve tous ces attraits d'un repos salutaire et bienveillant ${ }^{3}$.

Si ce n’était de la présence des mots « déconnecter» et «branché», bien de notre temps, il serait difficile de préciser à quand remonte la parution de la première réclame. Par son allusion à la douceur de la nature, à son potentiel libérateur, à la tranquillité et la possibilité de fuir la ville, cette publicité ressemble à s'y méprendre aux textes promotionnels publiés dans les journaux du début du siècle dernier. Notable, la similitude entre ces deux réclames atteste de la difficulté d'historiciser le rapport à la nature et d'en préciser la signification sociale et culturelle. Cela tient au caractère évasif de la nature, d'une certaine façon commune et banale parce qu'elle est omniprésente, mais aussi insaisissable par sa polysémie.

Dans un important recueil d'histoire environnementale sous sa direction, l'historien William Cronon discute des diverses significations données à la nature et des problèmes à saisir la "réalité » que recouvre le mot, souvent employé pour désigner l'ordre des choses, le caractère inéluctable

2. Les mots "déconnecter» et «branché» sont imprimés en caractère accentué dans le texte extrait du dépliant publicitaire suivant: «Découvrez un nouvel art de vivre dans les Cantons de l'Est», produit par le Cercle des Cantons, mars 2004.

3. «En villégiature», La Presse, 24 juillet 1916, 4. 
de certains phénomènes, ce qui va de soi ${ }^{4}$. Pourquoi donc se donner la peine d'étudier ce qui fait partie de l'entendement général? Considérée de façon globale, la nature est souvent comprise et perçue en opposition à la culture, comme essence ou réalité dénuée d'un contexte culturel. La vision de la nature réfère alors à la création et s'apparente à un phénomène qui se dérobe face à toute tentative de définition, à la manière dont Dieu, dans la tradition chrétienne, y échappe. Or, pour réelle que soit la nature saisie comme un équivalent du monde non humain, sa conceptualisation et ses définitions ne peuvent exister sans égard au contexte historique et culturel qui les met au jour et en définit les contours. Si l'entrée dans le Dictionnaire historique de la langue française stipule que ce terme désigne, depuis la fin du $\mathrm{xvII}^{\mathrm{e}}$ siècle, le "monde physique excluant $l^{\prime}$ homme et ses œuvres ${ }^{5}$ », force est de reconnaître que la nature "n'est pas une donnée en soi qui existerait de façon abstraite indépendamment des caractères des sociétés humaines ${ }^{6}{ }^{6}$. Refuge, nourriture, réservoir, oasis, éden, péril, incarnation du divin comme du diable: nombreux, les référents auxquels on associe la nature pourraient être multipliés à l'infini. C'est que ce mot, dont la puissance d'évocation est sans limites, ne livre pas lui-même son propre sens. Ses significations se construisent à travers les valeurs dont la nature est investie, les rêves qu'elle nourrit, les projets et les usages auxquels elle donne vie ${ }^{7}$.

Comme thème de recherche, la nature est surtout le lot des praticiens de l'histoire environnementale. Apparue ces dernières décennies pour tenter de saisir les rapports et les dynamiques entre l'homme et son milieu, l'histoire environnementale

4. William Cronon, «Introduction: In Search of Nature», dans W. Cronon, dir., Uncommon Ground. Rethinking the Human Place in Nature (New York, Norton, 1995), 23-56.

5. Dictionnaires Le Robert (Paris, 1998), 2348. Sur le développement de l'idée de nature à cette époque : Jean Ehrard, L'idée de nature en France dans la première moitié du XVIII siècle (Paris, SEVPEN, 1963).

6. Comme l'explique Patrick Fournier, mais en parlant de la pollution: «De la souillure à la pollution, un essai d'interprétation des origines de l'idée de pollution ", dans Christoph Bernhardt et Geneviève Massard-Guilbaud, dir., Le démon moderne. La pollution dans les sociétés urbaines et industrielles d'Europe/The Modern Demon. Pollution in Urban and Industrial European Societies (Clermont-Ferrand, Presses universitaires Blaise-Pascal, 2002), 55.

7. Une difficulté supplémentaire pour définir la nature tout comme historiciser ses manifestations provient du fait qu'elle ne génère pas d'archives comme telles, contrairement à d'autres objets d'étude: "Nature, like culture, as a story to tell, but there are so few sources of this history", Kristin Asdal, "The Problematic Nature of Nature: The Post-Constructivist Challenge to Environmental History", History and Theory, 42,4, (décembre 2003): 63. 
suppose $[\ldots]$ de renoncer au paradigme selon lequel l'homme se trouve en situation d'extériorité par rapport à la nature, et d'accepter l'idée de l'intégration de l'homme à la biosphère, des sociétés aux écosystèmes. Une telle vision nécessite la prise en compte des contraintes du milieu naturel (ce que les historiens ont fait, au moins en partie, depuis longtemps), mais aussi des bouleversements apportés (voire infligés) par l'homme à son environnement (ce qu'ils ont, pour l'essentiel, oublié $)^{8}$.

Ce champ de la discipline ne parvient cependant pas toujours à livrer les fruits promis, les ouvrages s'en réclamant ayant parfois du mal à bien démontrer ce qui fonde la spécificité de l'approche ${ }^{9}$. Ainsi, les travaux les plus anciens ont surtout cherché à documenter les interventions des humains sur la nature, entretenant l'illusion que l'environnement naturel pourrait être stable et capable de préserver son équilibre en autant que l'homme ne vienne pas perturber le cours "naturel» des choses. Une deuxième génération de travaux a néanmoins vu le jour qui cherche à exhumer les relations entre nature et société en considérant ces deux dimensions de manière dialectique et évolutive. Exemplaire est en ce sens le travail de Richard White sur le fleuve Columbia, défini comme une "organic machine ${ }^{10}$ ». Dans cette histoire des relations entre les humains et le fleuve, chaque partie de l'équation est prise en compte et les transformations qui en découlent de part et d'autre sont analysées. Comme le souligne à juste titre son auteur, le rapport des individus à la nature n'est jamais direct. Il est plutôt médiatisé par des représentations et des activités. $\mathrm{Si}$ au cours des premières décennies du $\mathrm{xx}^{\mathrm{e}}$ siècle, ce sont essentiellement les activités liées au travail, étudiées par White, qui conditionnent ce rapport, la pratique accrue de certains loisirs tels la chasse, la pêche, la randonnée ou la contemplation contribue aussi à faire évoluer les rapports à la nature tout comme ses représentations.

Les lignes suivantes scrutent certaines facettes de la villégiature dans la région montréalaise, plus spécifiquement en lien avec le développement des résidences secondaires, afin de mettre au jour les rapports à la nature de divers groupes de citadins. Intangible, la nature n'en constitue pas

8. Geneviève Massard-Guilbaud, "De la "part du milieu" à l'histoire de l'environnement", Le mouvement social, 200 (juillet-septembre 2002): 64-72.

9. La revue History and Theory a publié un excellent numéro qui offre un bilan des réflexions dans ce domaine: Brian Fay, dir., "Environment and History », 42,4 (décembre 2003). À lire aussi, l'essai de Ted Steinberg, "Down to Earth: Nature, Agency, and Power in History», American Historical Review, (juin 2002): 798-820.

10. Richard White, The Organic Machine: The Remaking of the Columbia River (New York, Hillard Wang, 1995). 
moins un outil ou un référent fondamental en tant que catégorie construite et utilisée aux fins de s'approprier certains espaces et d'y promouvoir et imprimer certaines valeurs. Comment les villégiateurs perçoiventils et définissent-ils leur environnement naturel? Comment s'y établissentils et quels usages en font-ils? Pour multiples que soient leurs définitions, les villégiateurs partagent une vision instrumentale de la nature, considérée comme un moyen pour se constituer des milieux de vie sur mesure, servant à l'affirmation de leur pouvoir et de leur prestige tout comme à la consolidation de leur identité sociale. Dans cet article, l'adoption d'une approche inspirée de l'histoire environnementale marque aussi la volonté de penser la ville dans une perspective englobant la campagne. De fait, toutes deux appartiennent au même milieu, et ce, en dépit des discours et des pratiques des villégiateurs qui, dans leur désir de se rapprocher de la nature, opèrent une coupure entre ces deux composantes ${ }^{11}$. La prise en compte de l'environnement constitue un angle, un point de vue de connaissance visant à éclairer le travail de remodelage effectué sur les milieux naturels pour les adapter à certaines fins et à cerner, en retour, comment les transformations de ces mêmes milieux se répercutent sur les représentations et les pratiques.

\section{LA PRESSE QUOTIDIENNE: UNE VITRINE DE LA VILLÉGIATURE ET DES VILLÉGIATEURS}

D’origine italienne, le terme de villégiature, sans équivalent en anglais, sert à désigner le séjour à la campagne ou dans un lieu de plaisance, pour se reposer et se divertir ${ }^{12}$. Il recoupe des réalités différentes selon les groupes sociaux en cause, les lieux et le type de séjour effectué. Dans le cas présent, j'étudie surtout les villégiateurs qui séjournent à la campagne à proximité de la ville, dans des résidences secondaires. Les données utilisées pour analyser ce mouvement ont été puisées dans quatre des principaux journaux,

11. Une coupure qui contribue à mystifier le fait que, loin de constituer des univers séparés, ville et campagne appartiennent au même environnement : «the urban and rural landscapes... are not two places but one. They created each other, they transformed each other's environments and economies, and they now depend on each other for their survival» : William Cronon, «Epilogue ", Nature's Metropolis. Chicago and the Great West (New York, W. W. Norton and Cy, 1991), 384.

12. C'est à partir des dernières décennies du $\mathrm{XIX}^{\mathrm{e}}$ siècle que le terme, désignant à l'origine le fait de séjourner à la campagne, se voit aussi associé à l'idée de vacances ou de récréation: André Rauch, «Les vacances et la nature revisitée (1830-1939)», dans Alain Corbin, dir., L'avènement des loisirs, 1850-1950 (Paris, Flammarion, 1995), 90 et ss. Sur l'idée de «re-création»/ «recreation», voir Cindy S. Aron, Working at Play: A History of Vacations in the United States (New York, Oxford University Press, 1999), en particulier l'introduction. 
deux anglophones et deux francophones, largement diffusés dans la région montréalaise et s'adressant d'abord aux habitants de cette dernière. En l'occurrence, La Presse, La Patrie, The Montreal Daily Star et The Montreal Gazette ont été systématiquement consultés durant les mois d'été, de mai à août inclusivement, de 1895 à 1910 , et de la même façon de 1916 jusqu'à la fin des années 1930, mais alors tous les trois ou quatre ans ${ }^{13}$. Ils comprennent des chroniques de villégiature, des articles sur certains de ses aspects, des illustrations ou des photographies, des poèmes, des bandes dessinées, des caricatures et des annonces publicitaires (figure 1).

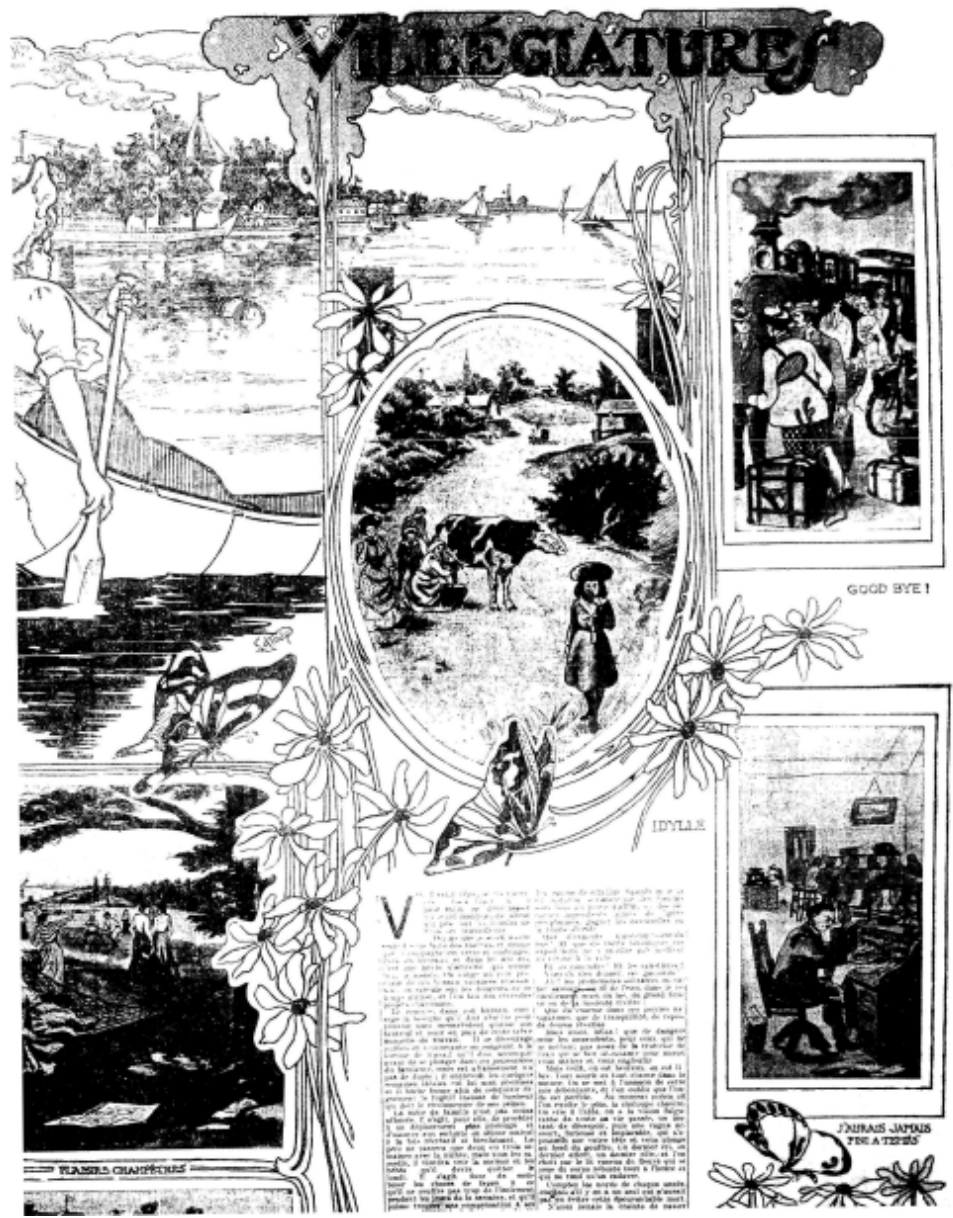

Figure 1: «Villégiature», La Presse, 4 juin 1904, p. 1

13. La recherche dans les journaux a été menée jusqu'à 1950 mais au vu de la diminution considérable des articles sur la villégiature durant la Deuxième Guerre mondiale et de la faible reprise de ceux-ci par la suite, je n'ai pas cru utile d'aborder cette dernière décennie. 
Durant la période allant de 1896 à 1910, la couverture journalistique de la villégiature est fort soutenue. Tous les quotidiens dépouillés y consacrent de trois à quatre pages dans leur édition du samedi, durant les mois d'été. Il arrive aussi que le sujet soit abordé à un autre moment de la semaine, mais cette couverture n'a pas fait l'objet d'un examen systématique. C'est The Montreal Daily Star qui, le premier, a établi cette pratique en 1895 , les autres journaux lui ayant emboîté le pas au tout début du $\mathrm{xx}^{\mathrm{e}}$ siècle ${ }^{14}$. Sur les centaines d'items, rencontrés dans ces journaux, nous en avons retenu plus de deux cents, répartis de façon à peu près égale au cours de la période, à l'exception des années comprises entre 1900 et 1907 où la couverture est la plus dense. Pratiquement tous les articles et les poèmes sont rehaussés d'illustrations de scènes de villégiature, à l'exception des chroniques qui, bien que coiffées d'une illustration à peu près toujours semblable, se déploient sur plusieurs colonnes. Les poèmes sont publiés plus fréquemment dans la presse anglophone que francophone. Il est aussi intéressant de noter que les caricatures ou bandes dessinées n'apparaissent qu’à partir de 1906, et ce, très irrégulièrement et uniquement dans le Montreal Daily Star.

Cette couverture dont la villégiature fait alors l'objet va contribuer à donner vie à ce phénomène et le rendre public, en particulier à travers des chroniques dont la particularité est d'être rédigées par les villégiateurs eux-mêmes. Ces chroniques informent sur leurs différents lieux d'élection, l'identité des individus qui s'y retrouvent, les activités offertes et leurs horaires, tout au long de la saison. Elles constituent une véritable vitrine permettant aux élites en villégiature d'exposer leurs faits et gestes aux yeux de tous les lecteurs, tout comme de se reconnaitre entre elles. Par le fait même, ces chroniques contribuent à la formation du groupe des villégiateurs et à l'élaboration de son identité sociale et culturelle. La villégiature, surtout avant la Première Guerre mondiale, constitue une composante fondamentale du processus de définition des élites montréalaises, alors aux prises avec des problèmes d'adaptation et d'ajustement, tout comme d'autres groupes sociaux, en cette période caractérisée par des changements intenses liés à l'urbanisation et l'industrialisation ${ }^{15}$.

14. On sait par des travaux menés sur diverses localités que la villégiature dans la région montréalaise se développe dès les premières décennies du XIX ${ }^{\mathrm{e}}$ siècle, même si celle-ci n'est traitée dans les journaux qu'à partir des années 1890. Il existe une foule d'histoires locales qui évoquent avec plus ou moins de détails la présence des villégiateurs dans la région montréalaise. Voir le rapport de recherche rédigé par Harold Bérubé, Les différents visages de Montréal. Plus près de la nature, l'ère de la villégiature (INRS-Urbanisation, Culture et Société, 2002), 33 p.

15. Les revues de l'époque abondent en articles qui décrivent par le menu les bienfaits découlant du rapprochement avec la nature, pour atténuer le stress provoqué par la vie moderne dans les villes: 
Des années 1890 à la Première Guerre mondiale, la couverture de la villégiature concerne essentiellement la région montréalaise, lieu d'élection des élites par excellence. Certains articles traitent bien d'autres destinations de vacances plus éloignées de la ville, mais ils sont moins nombreux et n'ont pas été retenus $\mathrm{ici}^{16}$. Cette couverture de la villégiature ralentit durant le conflit pour reprendre plus régulièrement une fois celuici terminé. Or la reprise des articles sur la villégiature s'accompagne d'une certaine évolution du phénomène. D’abord, les destinations des villégiateurs se diversifient. À la région montréalaise, toujours présente au moins jusqu'à la fin des années 1930, s'ajoutent notamment les Laurentides et les Cantons de l'Est. Au lieu d'être concentrée en quelques pages bien identifiées, la villégiature apparaît en différents endroits dans les journaux. De même, elle n'est plus systématiquement traitée dans chacune des éditions des samedis de l'été. The Montreal Daily Star est le journal qui aborde le plus fréquemment le sujet. Curieusement, cette couverture se tarit entre les années 1923 et 1929, dans La Presse et La Patrie. Nous avons recueilli autour d'une quarantaine d'items par année, composés d'un certain nombre d'articles, de chroniques, dont la présence demeure abondante, et d'une bonne quantité de caricatures et de bandes dessinées, qui ont pris la place jadis occupée par les illustrations. Les photos sont aussi nombreuses mais elles concernent surtout les lieux de villégiature les plus éloignés. Il n'y a plus de poèmes publiés, tandis que la publicité illustrée occupe désormais une place de choix. Elle représente autour de $40 \% \mathrm{du}$ corpus retenu pour l'analyse, composé de plus de 600 items.

Si elle a d'abord été amorcée par les élites, la pratique de la villégiature a aussi été suivie, après la Première Guerre mondiale, par des citadins aux

George Altmeyer, "Three Ideas of Nature in Canada, 1893-1914 ", Journal of Canadian Studies/Revue d'études canadiennes, 11,3 (août 1976): 22 et ss; Tina Loo, «Making a Modern Wilderness: Conserving Wildlife in Twentieth-Century Canada ", The Canadian Historical Review, 82,1 (mars 2001): 92-121; Patricia Jasen, Wild Things. Nature, Culture, and Tourism in Ontario 1790-1914 (Toronto, University of Toronto Press, 1995), en particulier chapitre 5. Dans la majorité des études, toutefois, c'est le penchant pour la nature sauvage qui a retenu l'attention des chercheurs. Sur la quête de la nature en milieu urbain ou proche de la ville: Michèle Dagenais, «Entre tradition et modernité. Espaces et temps de loisir à Montréal et Toronto au xx $x^{e}$ siècle », The Canadian Historical Review, 82,2 (juin 2001): 308-330; Caroline Aubin des Roches, Représentations et pratiques de la villégiature à Montréal au tournant $d u X X^{\mathrm{e}}$ siècle, mémoire de maîtrise (histoire), Université de Montréal, 2004.

16. Sur le phénomène de la villégiature au Québec et l'engouement ressenti à son égard par la bourgeoisie canadienne: Michel Lessard et Gaston Cadrin, «Les sentiers de la villégiature», Cap-aux-Diamants, 33 (printemps 1993): 10-13; Christian Auger, "À la recherche du pittoresque dans l'Estrie", Cap-aux-Diamants, 33 (printemps 1993): 59-62; Alan Metcalfe, Canada Learns to Play. The Emergence of Organized Sport, 1807-1914 (Toronto, McLelland and Stewart, 1987). Pour une analyse plus approfondie du phénomène: Cindy S. Aron, Working at Play..., op. cit. 
origines sociales plus modestes. Le type d'articles, les sujets abordés et la présence de plus en plus importante de bandes dessinées et de la publicité permettent de suivre les changements survenus dans le mouvement des résidences secondaires, à tout le moins dans certains de ses aspects. Une attention particulière sera donc accordée aux mutations en cours dans la composition sociale des villégiateurs mais aussi dans leur rapport à la nature, leurs lieux d'habitation et le type de résidences dans lesquelles ils s'installent. Abordées par les journaux dans des termes relativement abstraits et lyriques durant les années 1890-1910, ces diverses dimensions liées à la villégiature sont considérées, à partir des années 1920, sous des angles plus pratiques et en fonction de questions plus précises. La comparaison entre les deux périodes permettra de dégager certaines des composantes du modèle idéal des lieux de villégiature au cours de chacune d'entre elles et d'identifier les changements survenus. J'évaluerai enfin quels sont les aspects éventuellement transposés de la villégiature à la banlieue, dont la présence dans les journaux devient plus visible. De fait, avec le temps, la distinction entre villégiature et banlieue tend à s'estomper.

\section{LE TERRAIN DE JEU DES ÉLITES : L'ARCHIPEL DE MONTRÉAL}

La présence importante de l'eau et l'abondance de rives dans la région montréalaise, située au cœur d'un riche bassin hydrographique, ont attiré des générations de citadins, en quête de lieux de loisirs et éventuellement de vie, proches de la nature. Insérée dans un archipel formé de nombreuses îles dont les principales sont les îles de Montréal et Jésus, Montréal est entourée par les eaux de divers affluents: fleuve Saint-Laurent, lacs Saint-Louis et des Deux-Montagnes, rivière des Prairies ${ }^{17}$. Centrale dans l'histoire de Montréal, l'eau a joué un rôle déterminant dans son ascension comme métropole du Canada. Si les avantages économiques tirés de son environnement sont systématiquement soulignés dans les multiples études dont cette ville a fait l'objet tout au long de son histoire ${ }^{18}$, le rapport a été beaucoup plus rarement établi entre celui-ci et les activités de villégiature auxquelles il a pourtant donné lieu depuis le $\mathrm{xIx}^{\mathrm{e}}$ siècle $^{19}$. C'est seulement à partir du moment où la villégiature est devenue une activité

17. Gilles Sénécal et al., Le portrait environnemental de l'île de Montréal (Montréal, INRSUrbanisation, 2000), $19 \mathrm{p}$.

18. Harold Bérubé, Les différents visages de Montréal (INRS-Urbanisation, Culture et Société, 2002), $93 \mathrm{p}$.

19. Une exception notable est l'ouvrage de Marcel Samson, La résidence secondaire et la région métropolitaine de Montréal, thèse de Ph.D. (économie appliquée), Université de Droit, d'Économie et des Sciences d'Aix-Marseille, 1988. 
de masse, autour des années 1960 et 1970, qu'on a commencé à s'y intéresser plus systématiquement et à analyser ses impacts. À cette époque, pourtant, les pratiques de villégiature étaient déjà centenaires, les nombreuses rives et les collines des Montérégiennes ayant été prises d'assaut depuis longtemps par les élites montréalaises les plus fortunées.

La colonisation de la périphérie montréalaise par les villégiateurs prend véritablement son essor avec le développement des trains de banlieue ${ }^{20}$. À partir des années 1880, l'une après l'autre les lignes de chemin de fer de banlieue, suivant en partie le tracé des lignes ferroviaires commerciales établies quelques décennies auparavant, courent le long des rives de l'île de Montréal en plus de relier celle-ci à l'île Jésus et au continent en quelques endroits. Au cours de cette première phase, le développement de la villégiature dans la région va se faire en suivant des modèles de ségrégation sociale et ethnique relativement analogues à ceux inscrits dans le tissu montréalais, lui aussi segmenté ${ }^{21}$. Vers l'ouest de l'île et en particulier à Pointe-Claire, ce sont des membres de la communauté anglophone fortunés qui, d'abord comme estivants, vont s'y installer progressivement. Sur l'île Jésus, des villégiateurs d'origine francophone se transforment en quelques années à peine en banlieusards ${ }^{22}$. Sur le continent lui-même, face à Montréal, Boucherville représente un haut lieu de la villégiature des élites francophones ${ }^{23}$. Un peu plus à distance de Montréal, en allant cette fois vers le Nord, la région des Basses-Laurentides est elle aussi rapidement envahie par les villégiateurs. À Terrebonne Heights «sont piquetés des petits cottages britanniques et juifs", tandis que plus à l'ouest, des résidences d'été des francophones longent la rivière des Mille-Îles ${ }^{24}$.

Cette première vague d'explosion du territoire est suivie d'une seconde, à partir du début des années 1920, au moment où sont aménagés de nombreux axes routiers et plusieurs ponts tout autour de l'île. Comme l'illustre la carte plus bas, la multiplication des portes de sortie permet aux nouveaux automobilistes d'atteindre la région en de multiples

20. David B. Hanna, «Les réseaux de transport et leur rôle dans l'étalement urbain de Montréal», dans Horacio Capel et Paul-André Linteau, dir., Barcelona-Montréal. Desarrollo Urbano Comparado/Développement urbain comparé (Barcelona, Publicacions de la Universitat de Barcelona, 1998), 117-132.

21. Claire McNicoll, Montréal. Une société multiculturelle (Paris, Belin, 1993).

22. P. Dauphinais, L. Dauphinais et D. Marien, De la seigneurie à la banlieue : l'histoire de Lavaldes-Rapides des origines à la fusion (Montréal, sé, 1984).

23. Gilles Pepin, dir., La belle époque de la villégiature à Boucherville (Boucherville, Société d'histoire des Îles Percées, 1999), 36.

24. Serge Laurin, Histoire des Laurentides (Québec, Institut québécois de recherche sur la culture, 1995), 353 et ss. 
endroits et stimule encore davantage l'étalement urbain. Cette deuxième étape est aussi caractérisée par une diversification des origines sociales des villégiateurs. Le développement du réseau de tramways de banlieue, en cours d'achèvement à la veille de la Première Guerre mondiale, va permettre aux Montréalais d'origine plus modeste d'entreprendre à leur tour la colonisation de la périphérie ${ }^{25}$.

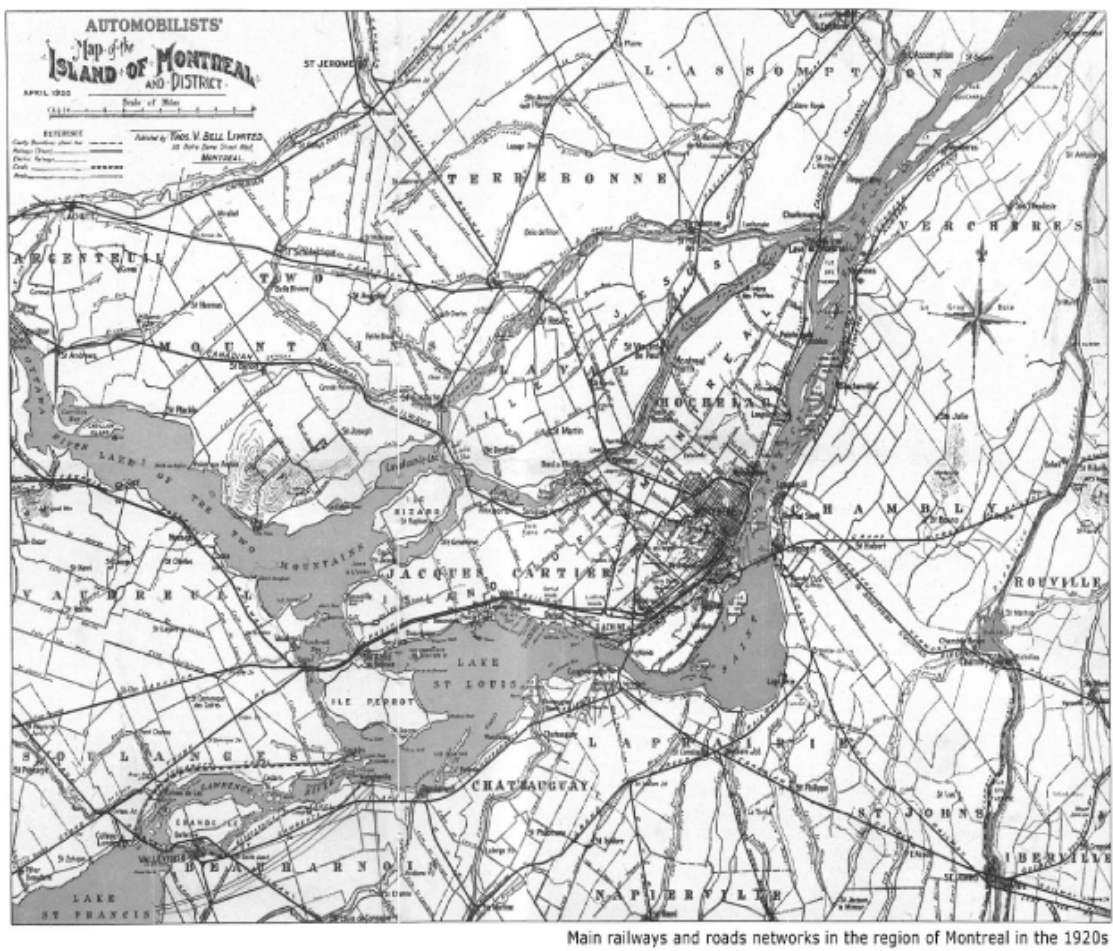

\section{L'APPEL DE LA NATURE}

Come to the woods, forget the street

Where traffic's noisy wheels are heard, And with those souls inspirers meet The murmuring pine and singing bird.
Let us forget the lines of care That on our faces deeply press, And breath a while the perfect air That longs to make our troubles less ${ }^{26}$.

Les dernières décennies du $\mathrm{XIx}^{\mathrm{e}}$ siècle correspondent à une période de grande (re)découverte chez les élites, celle de la nature et du plein air, rendus accessibles grâce au développement des moyens de transport et

25. David B. Hanna, "Les réseaux de transport... », loc. cit., 120-123.

26. "June Days at the Summer Resort», The Montreal Daily Star, 23 juin 1900, 10. 
en particulier du train, puis des tramways. Certaines études ont déjà documenté le rythme de croissance et les caractéristiques générales du phénomène de la villégiature dont les premières traces, pour la région montréalaise, remontent au début du $\mathrm{xIx}^{\mathrm{e}}$ siècle. Le plein air devient alors à la mode : "on vit au grand air, on s'en réjouit et on s'en vante...»; «Le goût du "dehors" avec ce que ce dehors comporte de mouvement, de liberté, d'énergie heureuse ${ }^{27} \ldots$... L'enthousiasme est grand, et les articles, nombreux sur le sujet, contribuent à l'engouement ressenti, en particulier pour la campagne: "Aux premiers sourires du printemps, aux premières chaudes œillades du soleil de mai, les habitants des villes n'ont qu'une pensée, s'évader de la fournaise des cités, du centre des affaires pour aller respirer, en toute liberté, les saines émanations des champs ${ }^{28}$.» (figure 2)

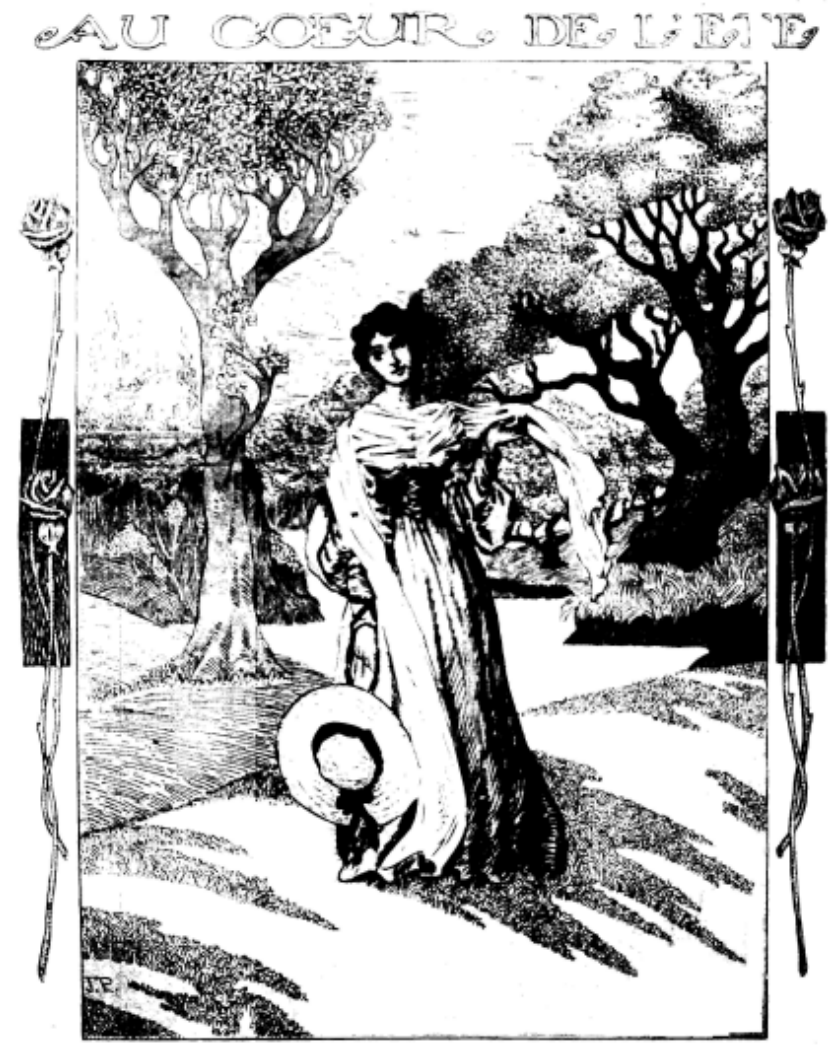

Figure 2: «Au cœur de l'été», La Patrie, 12 août 1905, p. 1.

27. «Le plein air», La Patrie, 13 août 1904, 15.

28. «À la recherche de l'ombre et de la fraîcheur. Nos endroits de villégiature - Comment on se prépare pour partir à la campagne - Des conseils utiles à tous », La Patrie, 16 mai 1905, 1-2. 
Cette campagne est généralement présentée comme une sorte de paradis terrestre, un éden, qui contraste nettement avec la ville, la première étant le lieu de la renaissance et de la régénération où la liberté, le mouvement, la détente deviennent possibles tandis que la seconde, celui d'un environnement malsain, de l'entassement et du stress lié à la vie quotidienne. Les bienfaits de la campagne ne sont donc pas que physiques: "This soul communion (with nature) does for the mental and spiritual side of our natures what food and clothing do for the physical. Without nourishment, without protection from the cold, our body would perish; without quiet thought, without time of introspection our souls $\mathrm{die}^{29}$. "Si cette conception spirituelle de la nature est particulièrement présente dans les discours tirés de la presse anglo-montréalaise, cela n'empêche pas la presse francophone de célébrer, elle aussi, les bienfaits de la nature quoiqu'en termes plus profanes. La nature et la campagne y sont ainsi considérées comme étant à la source «de la santé et d'une multitude de jouissances matérielles et de consolations morales ${ }^{30}$ ».

Mais cette campagne et cette nature, les villégiateurs ne se contentent pas de les célébrer. Ils souhaitent se les approprier et les acquérir, exprimant ainsi, pour certains, le goût pour «la possession du mont, du bois, de la plaine ${ }^{31}$ » ou pour d'autres, et de manière plus modeste, le désir pour «la possession d'un tout petit coin de terre où l'on pourra se construire un petit chalet d'été ${ }^{32}$ ». Partant, s'affirme aussi le besoin de modeler les lieux pour les rendre conformes aux idéaux, alors portés par ces élites urbaines. Le sentiment de respect et de vénération à l'égard de la nature, de sa beauté et de sa pureté se juxtapose au désir de la dompter et de l'adapter à ses besoins. Mieux, la capacité à modeler la nature est célébrée et associée au progrès et aux exploits technologiques propres à cette époque $^{33}$. Si les mille et un attributs de la nature offrent un horizon permettant de se libérer des tracas et des contraintes de la vie moderne, force est d'admettre que pour en profiter pleinement, il faut y apporter certaines

29. "At the summer resort», The Montreal Daily Star, 10 juin 1905. Sur la dimension religieuse du rapport à la nature et la campagne: Raymond Williams, The Country and the City (New York, Oxford University Press, 1973); Leo Marx, «Pastoral Ideals and City Troubles», Journal of General Education, 20 (janvier 1969): 251-271.

30. «Villégiature», The Montreal Daily Star, 2 juin 1906, 1-2.

31. «Le plein air», La Patrie, loc. cit.

32. «En villégiature», La Presse, 24 juin 1916, 4.

33. Les sentiments contradictoires à l'égard de la nature ne sont pas propres à cette période. En revanche, le sens ou la perception change selon le contexte. Sur cette époque en particulier: Rhona Richman Kenneally, "Depictions of Progress: Images of Montreal in Contemporary Guidebooks, 1839-1907», Journal de la Société d'étude de l'architecture au Canada, 23,1 (1998): 7-13. 
modifications et améliorations. Les villégiateurs ou les journalistes saluent et reconnaissent dans leurs articles le travail des élites urbaines dans les campagnes, qui ainsi "prennent un nouvel essor [...] s'embellissent $[\ldots]$ ». Et si à certains endroits "Les routes laissent encore à désirer", on admet que «tous les progrès ne peuvent se faire à la fois». Un des articles du genre insiste sur l'importance pour les agriculteurs et les habitants locaux, appelés en l'occurrence et de manière condescendante "nos amis des campagnes », "de bien entretenir leurs demeures, leurs bâtiments. Ce soin ne coûte rien. Mais comme la vie semble meilleure et plus gaie lorsque l'ordre et la propreté règnent sur nos fermes ${ }^{34}$ ! " Les villégiateurs s'adressent aussi aux « conseils municipaux de ces villes et ces villages [pour qu'ils] donnassent plus de soin et d'attention aux questions d'hygiène, à la propreté des rues, et à l'entretien des trottoirs ${ }^{35}$ ». Ce désir de se rapprocher de la nature se conjugue d'emblée avec la recherche de l'ordre et de la beauté, caractéristique des inclinations de la bourgeoisie anglo-montréalaise ${ }^{36}$.

Si elles constituent des sources potentielles d'enrichissement pour les populations déjà établies, l'installation des villégiateurs dans les environs de Montréal et la transformation concomitante des lieux provoquent leur lot de tensions. Bien que des articles soulignent régulièrement «le bienveillant accueil fait aux étrangers par les résidents et l'entente parfaite entre ceux-ci et ceux-là, dans l'organisation de fêtes dont l'unique but est de rendre à tous le temps de la vacance agréable ${ }^{37}$ ", la cohabitation n'est pas toujours harmonieuse, loin s'en faut. C'est qu'en se déplaçant, ces élites transportent avec elles leur conception de la nature et de l'environnement qu'elles souhaitent transposer dans leurs lieux d'élection. Il en résulte un véritable mouvement de colonisation de la périphérie par la ville au cours duquel sont disputés les usages des milieux naturels, tantôt liés à des pratiques de loisir et de récréation, tantôt à des activités écono-

34. "Les plaisirs de l'été», La Patrie, 29 juillet 1905, 10.

35. "Nos villégiatures", La Patrie, 20 mai 1905, 8. Une consultation rapide des règlements adoptés à cette époque par les municipalités ceinturant Montréal a permis de découvrir l'existence de nombre d'entre eux visant à contrôler et éliminer les «nuisances publiques» et à assainir les milieux locaux sur divers plans.

36. Brian Young, Une mort très digne. L'histoire du cimetière Mont-Royal (Montréal, McGillQueen's University Press, 2003), 15-16. Un penchant aussi partagé par les élites canadiennes-françaises en villégiature.

37. "Sault au Récollet», La Patrie, 15 août 1903, 9; «En villégiature», La Presse, loc. cit. Pour une représentation ironique des rapports entre villégiateurs et population rurale qui tranche avec la vision harmonieuse, dominante: «Summerings - Country Boarding and Hotelling, As a Cynic Sees It», The Montreal Daily Star, 28 juillet 1906, 7. 


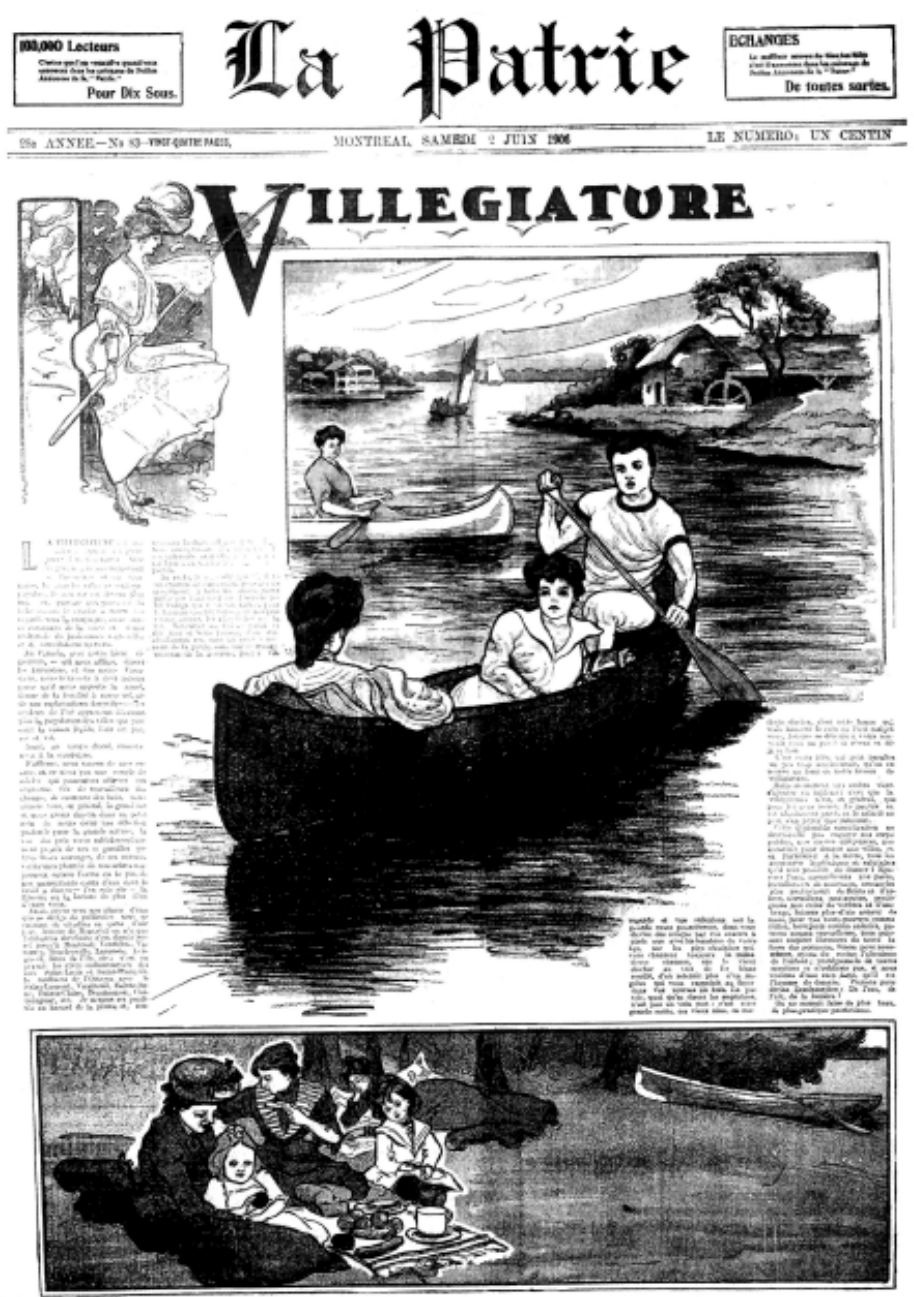

Figure 3: «Villégiature», La Patrie, 2 juin 1906, p. 1

miques de subsistance, comme dans le cas des agriculteurs notamment ${ }^{38}$. On ne trouve cependant pas trace de ces tensions dans les journaux dont le contenu, à cette époque, présente la villégiature sur un mode essentiellement optimiste voire triomphaliste.

38. Sur ces tensions: P. Dauphinais, L. Dauphinais et D. Marien, De la seigneurie à la banlieue..., op. cit., en particulier 71-83; Michèle Dagenais, "Returning to nature" : Vacation and Life Style in the Montreal Region", dans Geneviève Massard Guilbaud, Dieter Schott et Bill Luckin, dir., Resources of the City. Contributions to the European Modern Environmental History (Aldershot, Ashgate, 2005). 
Durant cette première période des années 1890 à la fin des années 1910, les articles dédiés aux aspects très concrets et pratiques de la villégiature sont rares en comparaison de ceux s'attardant à exposer les bienfaits des séjours à la campagne et du plein air. Les détails de la vie sociale des villégiateurs sont aussi nombreux qui rapportent parfois par le menu les activités organisées tantôt par les country clubs, tantôt par les boat clubs ${ }^{39}$. L'eau constitue un des éléments dont la présence est essentielle pour le regard, l'ambiance créée et la pratique de loisirs: «Ah! les promenades solitaires en canot sauvage [sic], au fil de l'eau, dans le recueillement muet du lac, du grand fleuve ou de la modeste rivière! Que de charme dans ces petites navigations, que de tranquillité, de repos, de douces rêveries ${ }^{40}$." (figure 3) L'eau donne aussi lieu à des activités sportives et compétitives élaborées, rapportées fidèlement dans les chroniques tout au long de l'été. Les régates se succèdent ainsi les unes aux autres, au fil des différentes localités de villégiateurs établies notamment sur les rives du lac SaintLouis, particulièrement prisées ${ }^{41}$. La pêche à la ligne est aussi populaire, présentée comme un art, "une œuvre de patience qui voue celui qui s'y livre à ses réflexions ». Or, il n'est pas donné à tous de pouvoir en goûter les joies : «les voluptés qu'éprouve le pêcheur à la ligne ne sont pas comprises du vulgaire: preuve qu'elles ne sont pas accessibles à tout le monde ${ }^{42}$ ». Mais l'eau est également source de danger et les conseils ne manquent pas pour prodiguer prudence et modération aux vacanciers.

La clôture de chaque saison estivale est aussi soulignée dans les chroniques de villégiature qui en profitent pour revenir sur les faits saillants de l'été et exposer les manières diverses des localités de marquer la fin des vacances. Le ton est souvent à la nostalgie :

As August draws to a close the gaiety of the summer season is on the wane. Very soon the migration of the summer visitors will begin... The healthy, hearthy country life must be exchanged for city ways, but tanned faces and

39. À côté des chroniques que publie sur une base hebdomadaire chaque quotidien, se trouvent aussi des articles expliquant les règles entourant l'organisation de réceptions d'été tels les pique-niques ou les «garden parties»: «Les réceptions à la campagne», La Presse, 29 juin 1907, 14.

40. "La natation», La Presse, 4 juin 1904, 1.

41. Alan Metcalfe a bien analysé le rôle des clubs sportifs comme pratique élitaire: Canada Learns to Play..., op. cit.; Caroline Aubin des Roches, Représentations et pratiques..., op. cit., 81-96. Sur le rôle des clubs dans l'aménagement de lieux exclusifs de villégiature: James M. Mayo, The American Country Club. Its Origins and Development (New Brunswick, Rutgers University Press, 1998).

42. «La pêche à la ligne », La Presse, 16 juillet 1904, 2. 
bright eyes will carry back to Montreal some of the freshness and sparkle of breezy Lake St. Louis, and many a pleasant memory of one of Pointe Claire jolliest seasons ${ }^{43}$.

De même, les chroniqueurs invitent les vacanciers à se retrouver l'année suivante, ce qui a pour effet d'accentuer le côté sélect en même temps que familier de ces lieux de villégiature, destinés exclusivement aux familles élues: "Already many are thinking of home, but at the same time are looking forward to next vacations with the hope of meeting every family face again, on the shores of Lake St. Louis ${ }^{44}$.»

\section{«ON AIME PLUS QUE JAMAIS LA CAMPAGNE ${ }^{45}$ »}

Si la présentation des bienfaits de la campagne, au cours de la première période, s'est souvent accompagnée du désir et de la nécessité de la modeler pour répondre aux besoins des villégiateurs, si la nature semblait rarement prête à l'emploi, les détails entourant son aménagement pour la rendre plus confortable et la doter de certains atouts de la ville moderne n'apparaissaient qu'en filigrane. On avait beau critiqué les routes parfois poussiéreuses dans certains endroits de villégiature, les dangers de l'eau ou encore la négligence de certains agriculteurs face à l'entretien de leurs bâtiments de ferme, les avantages semblaient nettement l'emporter sur les inconvénients. La vision bucolique de la nature ne disparaît pas soudainement avec la fin de cette première période, néanmoins présentée comme un âge d'or de la villégiature dans certaines monographies d'histoire locale ${ }^{46}$. À partir de la fin des années 1910, la douceur de la campagne est encore rappelée aux lecteurs çà et là, au détour d'un article. Cependant, la nature est de moins en moins présentée comme étant d'emblée parfaite et davantage comme perfectible. Les propos sur la campagne sont accompagnés de conseils destinés à l'aménager ou à améliorer l'environnement: «On aime plus que jamais le séjour à la campagne; dans le repos de la nature apaisante, les nerfs trouvent la détente... Plongé dans l'air vivifiant l'être se régénère, enrichit son sang, dilate ses poumons... Mais si l'être moderne réclame la vie de la nature, il entend bien de ne pas se priver du confort dont il a besoin ${ }^{47}$.» Le ton est donné. La campagne

43. "At the Summer Resorts", The Montreal Daily Star, 30 août 1902, 8.

44. Ibid.

45. «Pour rendre le séjour à la campagne agréable», La Presse, 5 août 1933, 20.

46. Notamment Marcel Paquette, Sainte-Rose: villégiature et tourisme, 1875-1950 (Laval, Éditions d'Antan, 1995); et Gilles Pepin, dir., La belle époque de la villégiature..., op. cit.

47. «Pour rendre son séjour...», loc. cit. 


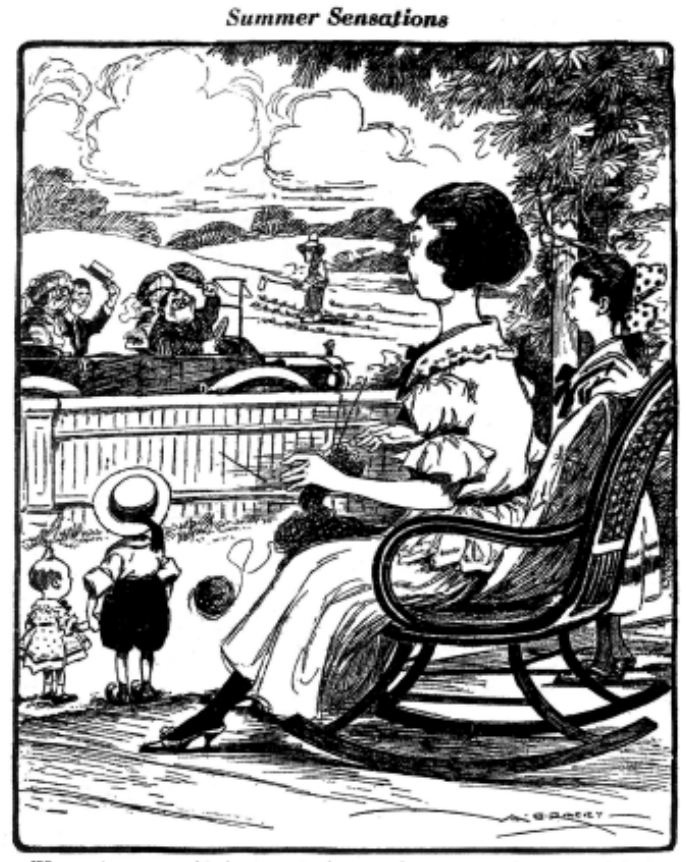

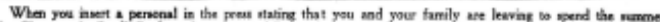

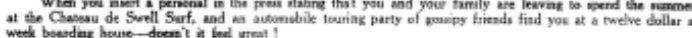

Figure 4: «Summer

Sensations », The Montreal Daily Star, 22 juillet 1922, p. 4
Figure 5 : «Prudence», La Presse, 27 juillet 1929, p. 17

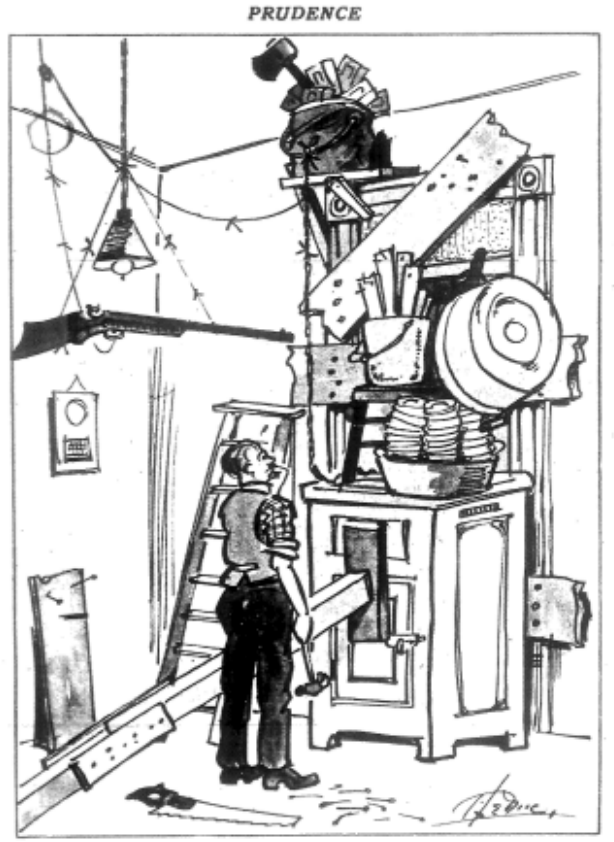

Leven qua veat deja fait cambrioler, of qui doit partir poesr la campagne. 
continue d'être recherchée et célébrée mais la vision des villégiateurs n'est plus tout à fait la même. Elle comporte, notamment, une bonne dose de pragmatisme.

Sans doute, le changement de ton découle en partie du choc causé par les pertes considérables liées à la Première Guerre mondiale. Il en résulte un certain désenchantement et la disparition quasi complète de la vision romantique et lyrique de la campagne, telle qu'elle s'exprimait auparavant. Les élites anglo-montréalaises sont particulièrement affectées par la perte de nombre de leurs descendants ${ }^{48}$. S'y ajoute l'élargissement graduel des rangs des villégiateurs dans la région montréalaise (figure 4). Plusieurs indices abondent en ce sens, au premier rang desquels le contenu même des articles de journaux et des publicités vantant les mérites de certaines propriétés à acquérir ou de certains endroits en insistant sur des attributs qui, manifestement, se rapportent à des villégiateurs dotés d'une moins grande aisance et indépendance financière ${ }^{49}$. Notable aussi est la présence croissante d'un discours ironique sur la villégiature qui en expose les vicissitudes et les désagréments. L'enthousiasme avec lequel on décrivait les bienfaits du plein air cède la place à des propos sur ses inconvénients, parfois pour mieux les contourner, à d'autres moments pour les déplorer (figure 5). De nombreuses caricatures et bandes dessinées rivalisent d'imagination et d'ironie pour évoquer le risque, pendant son séjour à la campagne, de se faire cambrioler à la ville; les conflits entre les résidants permanents et les villégiateurs; la difficulté de vivre avec les hordes de moustiques qui empoisonnent l'existence et irritent au plus haut point; l'épuisement des rivières de moins en moins riches en poissons; la pollution de certains lieux de baignade.

La campagne et ses saines émanations, les bienfaits illimités de la nature pour le corps et l'esprit ne semblent plus aussi évidents ni directement accessibles (figure 6). Si le grand air et la villégiature sont toujours prisés, il importe tout de même d'être prudent et de tester, au besoin, la qualité de l'environnement et des produits:

48. C'est ce qu'affirme Margaret Westley dans son ouvrage Grandeur et déclin. L'élite angloprotestante de Montréal, 1900-1950 (Montréal, Libre Expression, 1990).

49. Marcel Samson, La résidence secondaire..., op. cit., 74-75. Sur l'élargissement concomitant du tourisme aux classes moyennes: James Murton, "La "Normandie du Nouveau Monde": la société Canada Steamship Lines, l'antimodernisme et la promotion du Québec ancien", Revue d'histoire de l'Amérique française, 55,1 (été 2001): 3-44; de même que Cindy S. Aron, A History of Vacations..., op. cit. 


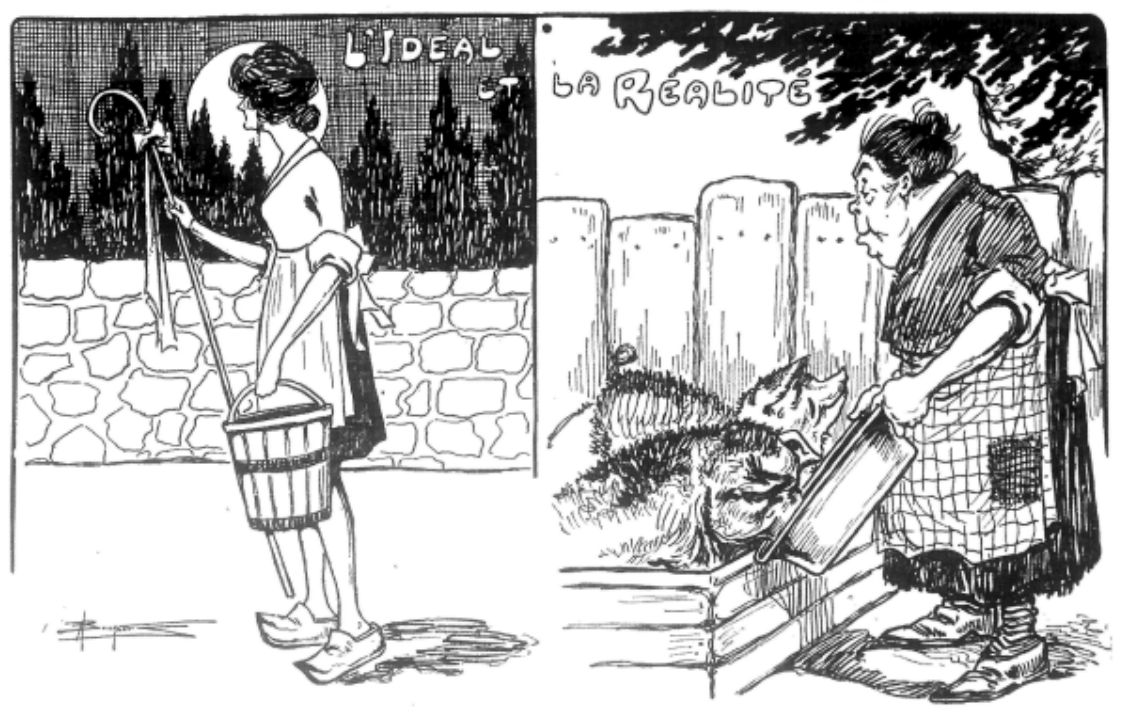

Figure 6: «L'idéal et la réalité», La Presse, 3 août 1929, p.33

There is no question but that a rest, a change in the environment, a period spent chiefly out of doors at play... are all good for everybody. [...] However it is foolish not to give some consideration to a few things, which, if overlooked, may result in the holidays developing the aspects of a calamity. Water in the country... will if contamined spread disease... just as readily as water in town or city. Milk in the country, despite popular belief, is generally not safe... You cannot tell from appearance whether or not cows have tuberculosis. [...] You cannot tell from appearance whether or not the farmer's well, the water which he uses is safe ${ }^{50} \ldots$

Le changement de ton est ici radical. La campagne n'est plus ce lieu divin et bienfaisant de jadis mais un endroit comportant des risques dont il faut apprendre à se méfier. Il faut dire que les découvertes survenues du côté de la bactériologie, depuis la fin du $\mathrm{XIX}^{\mathrm{e}}$ siècle, ont graduellement transformé la manière de percevoir l'environnement, disqualifiant la lecture empirique qui en avait jusque-là été faite au profit d'analyses menées en laboratoires pour en évaluer l'état ${ }^{51}$. On sait dès lors que la couleur et l'odeur de l'eau, notamment, ne suffisent pas à établir son innocuité. De même, le discours hygiéniste est beaucoup plus circonspect à l'endroit

50. "A Real Holliday", The Montreal Daily Star, 8 juin 1929, 26.

51. Denis Goulet et Othmar Keel, «Les hommes-relais de la bactériologie en territoire québécois et l'introduction de nouvelles pratiques diagnostiques et thérapeutiques (1890-1920)", Revue d'histoire de l'Amérique française, 46,3 (hiver 1993): 433-437 en particulier. 


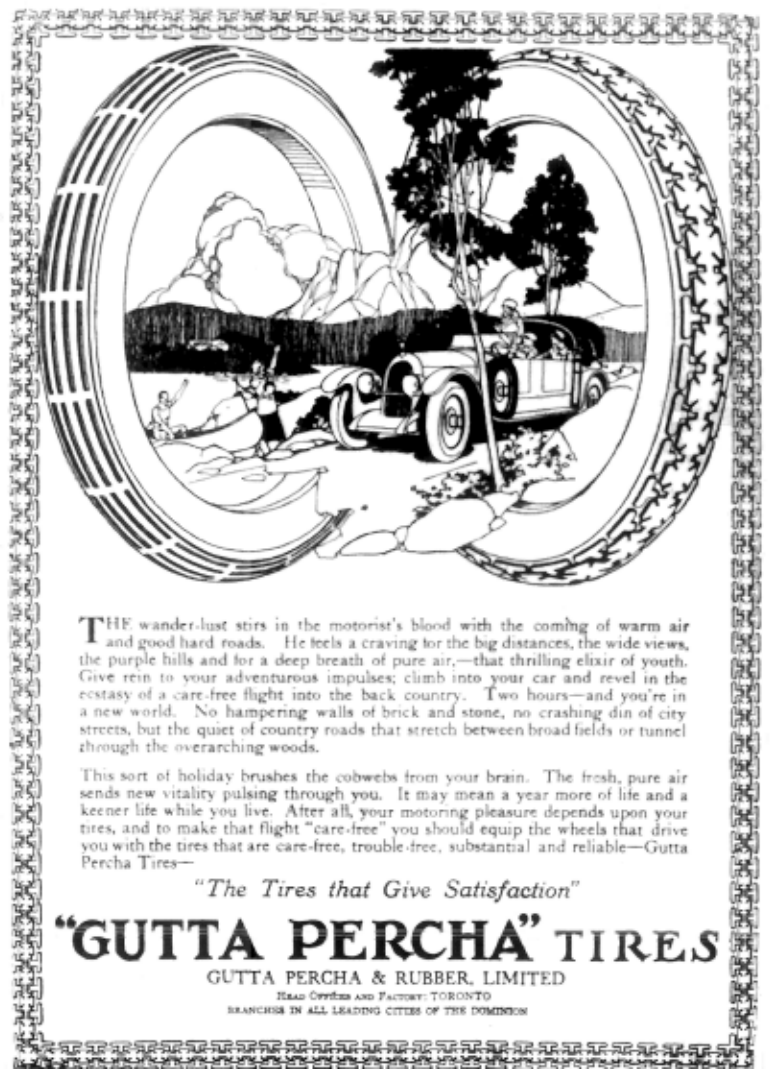

Figure 7: «Gutta Percha Tires», The Montreal Gazette, 8 mai 1920, p. 19

des séjours à la campagne comme moyen de rétablir la santé ${ }^{52}$. Une autre des raisons pour laquelle le discours sur la nature se modifie tient peutêtre, en outre, à la dégradation des milieux naturels, causée par la pollution de certains lieux, la transformation voire la surexploitation d'autres (figure 7). Ce changement de ton n'empêche pas la manifestation d'un sens de supériorité ou de propriété vis-à-vis de la nature ou de la campagne, perceptible dans de nombreux articles et visible au détour de certaines publicités, particulièrement celles faisant la promotion de marques d'automobiles ${ }^{53}$. Mais ce discours n'est plus aussi triomphant et

52. Comme le précise éloquemment le passage suivant, tiré du Bulletin sanitaire: «Les personnes qui vont en villégiature $[\ldots]$ doivent prendre des précautions auxquelles on ne pense guère lorsqu'on reste dans les villes »: «La fièvre typhoïde en été », Bulletin sanitaire, 26,3 (1926): 92-93. Voir aussi «L'hygiène à la campagne pendant l'été», Bulletin sanitaire, 24,2 (1924): 50-53.

53. "The wander-lust stirs in the motorist's blood with the coming of warm air and good hard roads. He feels a craving for the big distances, the wide views, the purple hills and for a deep 


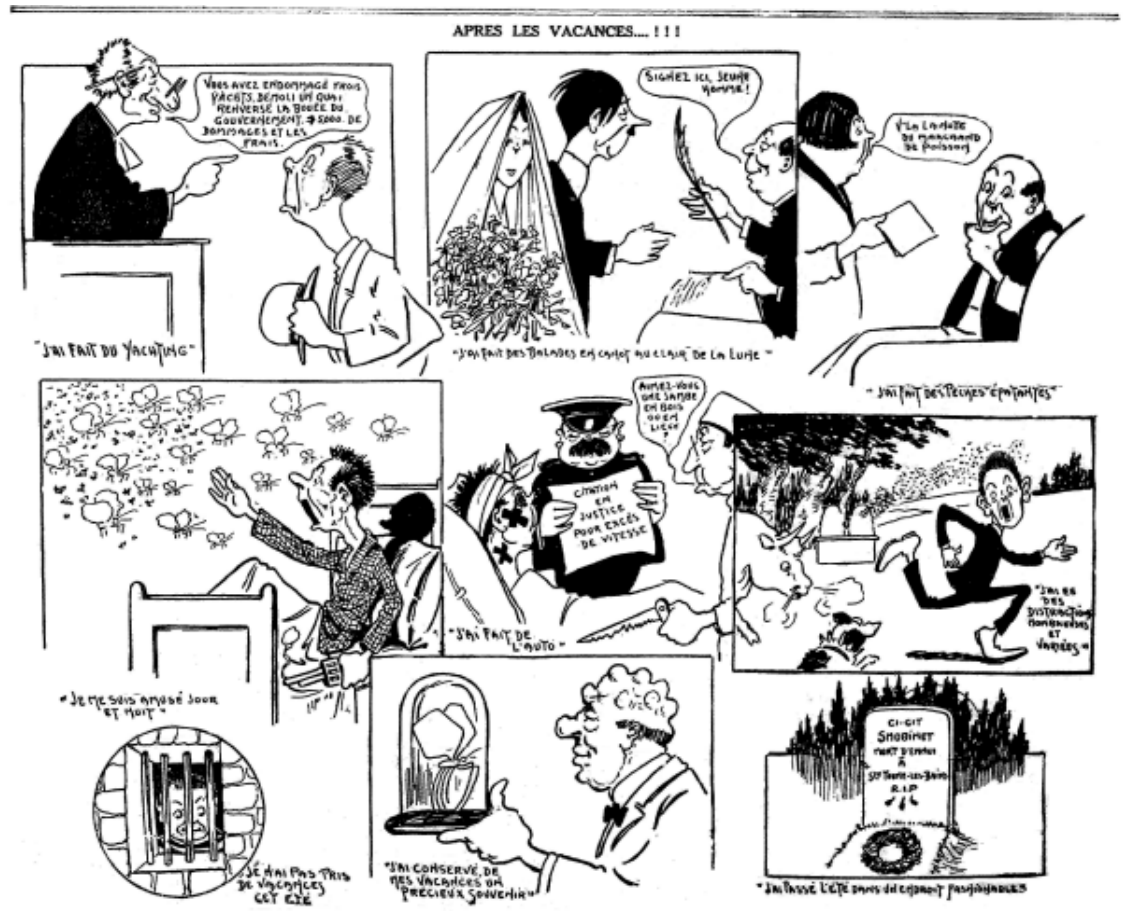

Figure 8: «Après les vacances...!!!", La Presse, 24 août 1929, p. 49.

surtout, il côtoie les propos désenchantés et ironiques précédemment exposés (figure 8).

\section{« VOUS VOULEZ LA SANTÉ ET LE BONHEUR? HÂTEZ-VOUS D'ACHETER L'UN DE CES LOTS ${ }^{54}$."}

Mais quels sont alors les ingrédients nécessaires au bonheur et à la santé? C'est d'abord de pouvoir s'offrir une maison à la campagne, tantôt appelée villa, tantôt cottage, tantôt chalet ou camp, plus modestement ${ }^{55}$. La proxi-

breath of pure air, - that trilling elixir of youth. Give rein to your adventurous impulses; climb into your car and revel in the ecstasy of care-free flight into back country»: "Gutta Percha Tires », The Montreal Gazette, 8 mai 1920, 19; "More for the Money; New Dodge Brothers Six", Idem, 4 mai 1929, 16; «In Performance As in the Style the Finest Oakland Ever Built», The Montreal Daily Star, 27 juillet 1929, 29.

54. "Canotage, pêche et yachting», La Patrie, 28 juillet 1923, 23.

55. L'importance de la maison dans l'expérience de la villégiature, en particulier dans la tradition anglaise, mériterait d'être développée plus avant. Sur cette question: Sophie Cueille, «La campagne à portée de jardin. L'apport des sociétés d'horticulture au xix ${ }^{\mathrm{e}}$ siècle », Histoire urbaine, 8 (décembre 2003): 134-135 en particulier; Witold Rybczynski, Waiting for the Weekend (New York, Viking, 1991), chapitre 7; Karen M. Fraser et Christine O’Malley, «Building for the Outdoors: 
mité des lieux de villégiature avec la ville et la facilité à s'y rendre en automobile ou par le train sont aussi à mettre au compte des avantages à considérer. La présence de l'eau, mieux son accès direct, est toujours fort prisée comme le démontre le texte de cette publicité, parmi d'autres: "The coming high-class, exclusive Summer Resort. Just put on the market; occupies on of the most beautiful spots in Canada - fronting on Lake StLouis and the Ottawa River ${ }^{56}$; ou celui de cet article: «Le plus beau plaisir qui puisse s'offrir à celui qui a les moyens de se construire une propriété pas très loin de la ville c'est bien celui de la pêche à la ligne. Ce plaisir peut facilement être obtenu par tous ceux qui ont leur villa près de Rivière-des-Prairies ${ }^{57}$. "Un autre ingrédient du bonheur, c'est de s'établir sur un terrain suffisamment vaste pour contenir des «flower beds, vegetable gardens, fruit orchards...» sans pour autant être complètement isolé, car cela pourrait s'avérer insécurisant: "Valois est sûrement un des endroits les plus pittoresques... de très jolis cottages sont groupés, tout en observant la distance qu'il faut pour jouir du repos et du quasi-isolement que tous les citadins vont chercher à la campagne ${ }^{58}$.» Cette référence à la nécessité de s'installer sur un lot suffisamment éloigné des voisins, sans l'être tout à fait, est nouvelle par rapport à la période précédente; elle rappelle la disposition des maisons dans les banlieues pavillonnaires, séparées les unes des autres par des jardins.

Insérées parmi les petites annonces des maisons à vendre, les publicités sur les développements suburbains reprennent d'ailleurs les mêmes éléments pour faire valoir leurs mérites respectifs: «Crawford Park [...] offers many avantages over most of the subdivisions on and around the Island of Montreal, its easy access [...] its ideal location on the shore of the St. Lawrence, where the air is pure, its modern improvements, etc. ${ }^{59}$.» Que dire de la ville de Hampstead, limitrophe, elle aussi, de Montréal:

Two Early Twentieth-Century Kingston-Area Cottages», Bulletin de la Société d'étude de l'architecture au Canada, 18,3-4 (septembre-décembre 1993): 73-78; pour une perspective plus proprement architecturale de la maison de campagne, conçue pour s'harmoniser à l'environnement global et y participant: France Gagnon-Pratte, Maisons de campagne des Montréalais, 1892-1924 : l'architecture des frères Maxwell (Montréal, éditions du Méridien, 1987); pour une déclinaison du thème de la maison dans le parc, en plus modeste mais néanmoins cossue: Brian R. Matthews, A History of Pointe-Claire (Pointe-Claire, Brianor Ltd, 1985), chapitre 12 en particulier sur les secteurs "Cedar Park» et "Bowling Green» de cette localité.

56. "Isle Perrot", The Montreal Daily Star, 12 août 1922, 24.

57. "Canotage, pêche et le yachting", La Patrie, 28 juillet 1923, 23.

58. "Valois, plage très courue des citadins", La Patrie, 15 juin 1918, 16.

59. "Crawford Park (Verdun)", The Montreal Daily Star, 20 mai 1922, 32. 


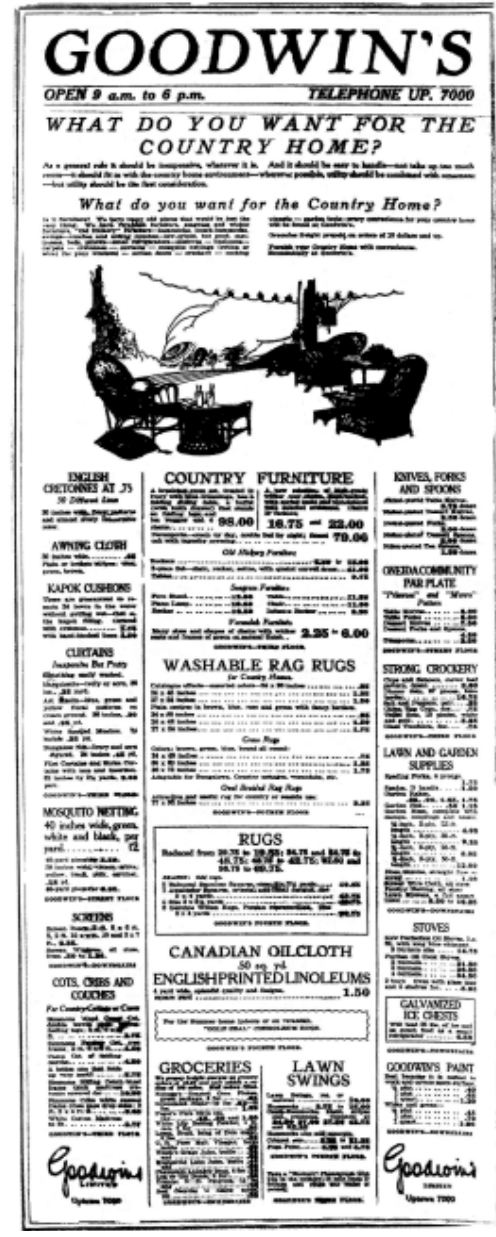

Figure 9: «What do you want for the country home? ", Montreal Daily Star, 20 mai 1922, p.44
Here's the ideal place to live... "where life is better"... we say "happy Hampstead» because here lawns are broad and smilling, home are attractive and appealing and here is quiet and rest away from the rush and turmoil of the big city... here... you do not merely reside - you «live»... the Hamsptead golf course is a healthy five minutes walk from your front door. A highly restricted community ${ }^{60} \ldots$

Si les localités dont on vante ainsi les mérites sont moins huppées que celles où se sont développés les endroits de villégiature typiques de la première période, elles constituent néanmoins des lieux relativement sélects et prisés, à cette époque comme aujourd'hui.

À partir des années 1920, la «maison de campagne idéale ${ }^{61}$ " est susceptible de comporter un revêtement en «stuc, à l'architecture délicate, d'un style original, offr[ant] des volets ouvragés aux fenêtres de l'avant, sa terrasse, sa lourde porte à panneaux au-dessus de laquelle est suspendue une lanterne d'un modèle antique». Il s'agit là de tout un ensemble de «caractères qui peuvent la faire distinguer parmi un grand nombre d'habitations de villégiature et même parmi nombre des maisons de ville».

En substance, on retrouve ici deux des qualités recherchées: une certaine exclusivité mais aussi un type de maison qui soutient la comparaison avec les maisons de ville puisqu'elle est équipée de tout le confort moderne. Or ce confort ne saurait surgir comme par enchantement. Il importe au villégiateur de se procurer tout le matériel et le mobilier requis:

60. "Hampstead, Homes of Distinction at Low Cost», The Montreal Daily Star, 17 août $1929,28$.

61. "À la conquête d'un chez soi. Une maison de campagne idéale », La Presse, 5 juin 1926, 35. 


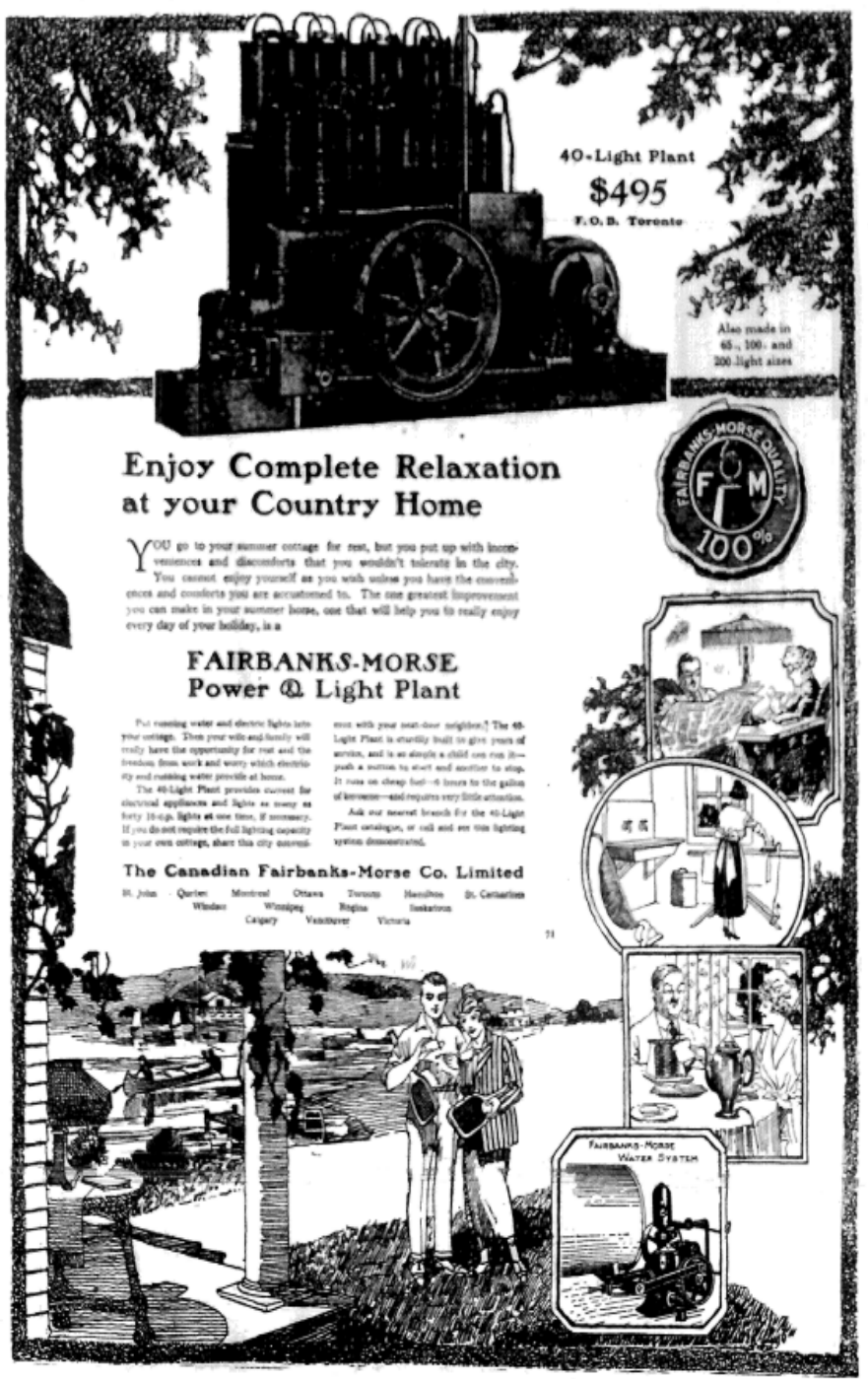

Figure 10: «Enjoy complete relaxation at your country house», The Montreal Gazette, 8 mai 1920, p. 8

il lui faut de bons fauteuils pour se reposer, à portée de sa main des tables, une bibliothèque, des tabourets, des coussins: les femmes veulent avoir leur table à ouvrage, les messieurs, leur table de fumeurs, tous la tablé à thé et la table de bridge. À ces meubles indispensables [...] se doit joindre un "abri» qui permette à temps de se défendre contre le vent, le soleil, la pluie ${ }^{62}$. 
Signe des temps, le mobilier doit être pratique, ni lourd ni encombrant, et bien s'intégrer à l'environnement naturel: «it should fit in the country home environment ${ }^{63} \ldots$ » (figure 9). Mais par-dessus tout "utility should be the first consideration". Ainsi, pour profiter de la campagne, il importe de s'aménager un petit coin de paradis doté de tout le confort et des avantages auxquels on est habitué: «You cannot enjoy yourself as you wish unless you have the conveniences and comfort you are accustomed to [...] Put running water and electric lights into your cottage. Then your wife and family will really have the opportunity for rest and the freedom from work and worry which electricity and running water provide at home ${ }^{64}$.» (figure 10) Sans compter la foule d'accessoires destinés, eux aussi, à rendre la vie à la campagne agréable: un phonographe portatif, une trousse de couture, de la papeterie spéciale «to give a final touch of distinction to the appointment of your summer home ${ }^{65}$ ». Et pourquoi pas apporter «an organ with you to your summer cottage and leave it there for future seasons ${ }^{66} »$ ? Aussi contradictoire puisse-t-elle apparaître, cette quête de la nature qui ne veut en rien céder au confort de la ville est bien de son temps. Elle s'apparente au courant ou à l'état d'esprit antimoderniste, encore manifeste dans les années 1920 et 1930. Caractéristique en est la recherche de l'authentique, du traditionnel et du naturel en signe d'un certain refus de la modernisation. Mais contrairement à ce qui a été observé pour le tournant $\mathrm{du} \mathrm{xx}^{\mathrm{e}}$ siècle, cette recherche ne vise plus à critiquer la modernité ou à tenter de la réformer et témoigne plutôt d'une attitude disposée à s'en accommoder ${ }^{67}$.

\section{LA VILLE À LA CAMPAGNE OU LA CAMPAGNE À LA VILLE?}

Pourtant mues par le désir de se rapprocher de la nature et de la campagne et, par conséquent, de s'éloigner de la ville, les pratiques de villégiature donnent ainsi lieu à une interpénétration de plus en plus marquée des deux univers. Celle-ci s'exprime aussi à travers tous les services de livraison, mis sur pied par les fournisseurs montréalais. Marchands d'alcool ${ }^{68}$,

63. "What Do You Want for the Country Home?», The Montreal Daily Star, 22 mai 1922, 44.

64. «Enjoy Complete Relaxation at Your Country House. Fairbanks-Morse Power \& Light Plant », The Montreal Gazette, 8 mai 1920, 8.

65. Se référer aux petites annonces classées des divers journaux, notamment: The Montreal Daily Star, 22 juin 1918, 6, et 20 juillet 1918, 32; The Montreal Gazette, 4 juin 1921, 2; The Montreal Gazette, 9 août 1941, 17.

66. The Montreal Daily Star, 17 juin 1922.

67. Pour une excellente discussion sur l'antimodernisme tel que vécu par les élites au-delà de ses premiers balbutiements: James Murton, «La "Normandie du Nouveau Monde”... », loc. cit.

68. "Make Sure of Your Holiday Liquor Needs. Place Your Order at Once and Be Sure of an Adequate Supply While You Are in the Country ", The Montreal Daily Star, 22 juin 1918, 6. 
librairies, épiceries, pharmacies et grands magasins offrent aux villégiateurs la possibilité de se faire livrer des marchandises en tout genre, durant tout le temps de leurs séjours à la campagne: «We now offer our country-resident clientele a NEW SERVICE, whereby they can order their groceries, which will be properly packed and shipped direct to their summer homes. Regardless of where your cottage may be located, just leave a list of your requirements with the store management and he will do the rest $^{69}$.» Quelle meilleure façon existe-t-il d'atténuer les affres du dépaysement ou les inconvénients résultant du manque de choix, caractéristique des petites épiceries des campagnes et des villages? Mais ce qui est présenté comme le summum du confort en villégiature, parce que permettant d'y reproduire ses habi-

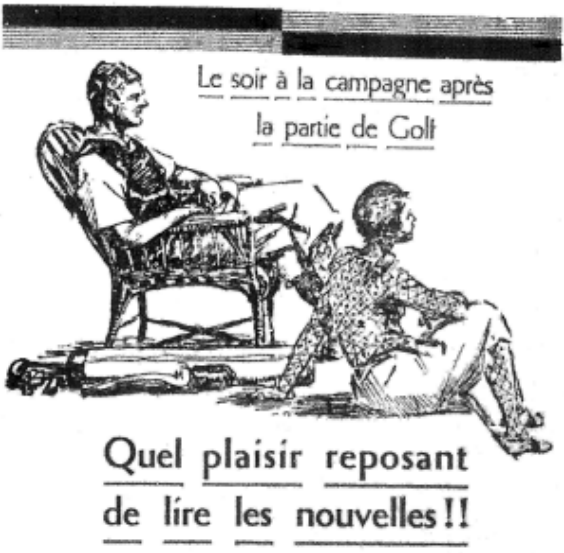

Satisfaction bien douce en villégiature, que d’oublier les tracas, la vie intense et artificielle de la ville... Mais demi-satisfaction si l'on est séparé complètement de nos habitudes et de ce quil nous intéresse ; nouvelles de sport, de la finance, de politique locale, nationale ou étrangéré, nouvelles de notre ville, nouvelles du monde entier... Nous sommes habitués à être renseignés, et vraiment il manque quelque chose à la villégiature, si nous ne sommes pas constamment tenus, par la "Presse," au courant des nouvelles et des évenements qui nous intéressent,

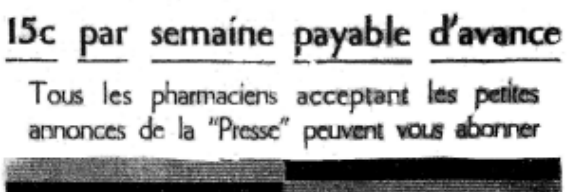

Figure 11: «Quel plaisir reposant de lire les nouvelles!!», La Presse, 10 juin 1933, p. 45 tudes quotidiennes sans les inconvénients et le stress qui y sont liés, c'est la livraison de son journal favori. Comme le stipule éloquemment la réclame de La Presse (figure 11):

Satisfaction bien douce en villégiature, que d'oublier les tracas, la vie intense et artificielle de la ville [...] Mais demi-satisfaction si l'on est séparé complètement de nos habitudes et de ce qui nous intéresse: nouvelles du sport, de la finance, de politique locale, nationale ou étrangère, nouvelles de notre ville, nouvelles du monde entier... Nous sommes habitués à être renseignés, et vraiment il manque quelque chose à la villégiature, si nous ne sommes pas constamment tenus, par la "Presse», au courant des nouvelles qui nous intéressent ${ }^{70}$.

69. «What... Groceries at City Prices...!!", The Montreal Gazette, 22 juin 1933, 3.

70. "Quel plaisir reposant de lire les nouvelles!!», La Presse, 10 juin 1933, 45. 
Cette interpénétration de plus en plus importante de la ville et de la campagne est aussi manifeste dans les publicités des grands magasins qui offrent aux consommateurs, et surtout aux consommatrices, une gamme étendue de produits pour embellir l'intérieur tout comme l'extérieur de leur maison; "get out of doors - enjoy the wonderful weather - and brighten up the porch or the verandah with gay new furniture... Even if you have to spend the Summer in town - you can do it in comfort and create the illusion - if not the reality - of perfect leisure ${ }^{71}$ ! Pour se donner ainsi l'illusion de la campagne à la ville et se mettre dans l'esprit des vacances, il faut évidemment avoir à sa disposition un jardin ou une cour arrière, ce que seuls les pavillons de banlieue offrent ${ }^{72}$. Ne retrouvet-on pas en substance une adaptation en plus modeste du modèle bourgeois de la maison de campagne intégrant la nature environnante à défaut de pouvoir s'y intégrer?

Le caractère commercial de la villégiature s'est donc nettement accentué au cours de la deuxième période. Il est certain que cet état de fait tient en partie aux sources utilisées, notamment à la publicité, beaucoup plus présente dans les journaux à partir des années 1920. De même, la caricature, surtout de nature politique au début du siècle, s'oriente vers des sujets à caractère plus social, avec le temps. Sans compter la présence plus considérable des bandes dessinées et la diminution concomitante des chroniques de villégiature, au cours des années 1930. Mais pour être sans aucun doute un peu trompeurs, ces développements n'en constituent pas moins des produits et des témoins de leur temps qui traduisent les tendances et les préoccupations de l'époque dans laquelle ils s'inscrivent. Si la présence plus directe et visible d'objets et de considérations matérielles induit un autre type de rapport à la nature, il n'est pas pour autant dénué de toute forme de référence à son caractère mystique et moral comme en témoigne le billet suivant, dont la présence se fait encore sentir dans les journaux à la fin des années 1930 :

A sense of escape comes with the hollidays. It is felt with varying intensity, but it is the hard worker of hand or brain to whom it comes with the full force of its strongest reactions...

Rest is never given except to those who have won it by true and honest work done with all their power.

71. "Make the Most of SUMMER!», The Montreal Gazette, 20 mai 1933, 3.

72. Voir aussi cette autre publicité qui annonce un solde d'articles destinés spécifiquement à l'embellissement de la cour arrière: «Backyard Day at Ogilvy's», The Montreal Daily Star, 6 mai 1933, 9. 
Only in quietness, which is the condition of spiritual receptivity do men learn the true secret of life. Great souls have always found their inspiration in quietness. [...] They have sought it far from the crowded city and the noise of the market ${ }^{73}$.

Ce type de discours illustre également comment la construction sociale de la nature puise à un ensemble de références dont certaines, même si elles sont plus anciennes, s'immiscent dans les représentations plus contemporaines. Il rappelle enfin la tension inhérente à la notion de loisir moderne qui, tout en valorisant le repos sain, réparateur et ressourçant, le loisir utile et bienfaiteur, ne manque jamais de les opposer aux mauvais loisirs et à la crainte de l'oisiveté et du désœuvrement.

\section{CONCLUSION}

Une des principales dynamiques à l'origine du processus de suburbanisation dans la région montréalaise, la villégiature, a bouleversé les milieux environnant Montréal et les a transformés de manière importante. L'examen de la presse montréalaise durant la saison estivale a révélé comment certaines des pratiques des villégiateurs ont conduit à urbaniser la région, en modifiant graduellement ce territoire, d'abord occupé par l'agriculture, en une vaste banlieue. S'il peut paraître paradoxal que la quête de la nature ait provoqué l'urbanisation de la campagne, cette contradiction n'est que superficielle. Même si elle exprime un désir de distanciation de la ville, la villégiature constitue un phénomène fondamentalement citadin et moderne, en phase avec le processus d'urbanisation et les avancées technologiques de la seconde moitié du $\mathrm{xIx}^{\mathrm{e}}$ siècle. En fuyant la société urbaine et industrielle pour se rapprocher de la nature, les élites ont favorisé la transformation de l'environnement et un re-modelage des paysages dans la mesure où elles ont transporté avec elles et imposé leur vision de la nature, leurs pratiques sociales et leurs activités de loisir. Plutôt que de réaliser un simple changement de décor, les élites ont donc travaillé à adapter les milieux convoités en fonction de leurs aspirations.

Inscrit au cœur des pratiques des villégiateurs et de leur rapport à la nature se trouvent le désir de s'éloigner de la ville tout comme le besoin d'y demeurer lié, du moins par certains aspects. Aussi bien au cours de la première que de la seconde périodes examinées, la tension semble constante entre la quête du dépaysement à laquelle aspirent les villégiateurs et l'envie de se recréer dans un cadre qui leur est familier. Le travail effectué dans 
l'environnement rural, particulièrement au cours de la première période, par la construction de résidences et l'installation de commodités et de services publics en témoigne, tout comme la constitution d'une vie sociale intense par l'intermédiaire des divers clubs et associations, des compétitions sportives et des événements mondains. L'une et l'autre pratiques représentent des manières de conserver présent et vivant le lien avec l'univers matériel, social et culturel urbain. Au cours de la deuxième période, plus marquée par un certain désenchantement, le travail de raccordement à la ville apparaît davantage orienté en direction de la maison comme telle, de son installation et de l'acquisition de biens matériels, de manière à rendre la vie en villégiature agréable. Que ce soit au cours de la première ou de la seconde périodes, force est de reconnaître l'existence d'un continuum villecampagne qui, en dépit de discours cherchant à distinguer l'un et l'autre de ces milieux, se réalise néanmoins à travers les individus eux-mêmes, leurs représentations, les paysages remodelés, les maisons et les objets, ou encore les pratiques sociales et culturelles.

Or, si les efforts déployés pour accommoder la campagne passent par l'importation de critères de confort propres à la ville, rendant l'un et l'autre milieux de plus en plus semblables, il est frappant de constater à quel point le désir de rapprochement avec la nature demeure au cœur des aspirations et des pratiques. Même instrumentalisée et transformée comme elle le devient, la nature, ou plutôt l'idée de nature, constitue effectivement l'ingrédient de base de la villégiature à chaque période, tout comme elle représente une donnée fondamentale du modèle bourgeois de la banlieue. En substance, se trouve révélé le double caractère de l'idée de nature: à la représentation a-historique qui transcende périodes, milieux de vie et groupes sociaux, se greffe une conception plurielle, ancrée dans un temps et un espace donnés, comme en témoigne la transformation du contenu des discours sur la nature, énoncés au fil du temps. C'est cette polysémie de la nature tout comme la multitude des possibles auxquels les différents acteurs l'associent qui compliquent toute tentative visant à la définir. Elle n'en constitue pas moins l'ingrédient fondamental, le moyen tout comme la justification par le biais desquels une classe sociale peut élaborer le mode de vie qui lui convient. Les valeurs telles la liberté, la pureté, la fluidité, le caractère sacré qui sont accolées à la nature fondent et alimentent le désir de s'en approprier et de se constituer, très concrètement, des milieux de vie exclusifs.

La nature ou la présence d'éléments naturels, l'espace, les commodités, les équipements de loisirs, le cachet des maisons, mais aussi le caractère 
exclusif des milieux, le sentiment d'indépendance et de sécurité que confère la propriété familiale, le confort moderne: tous ces éléments ne constituent-ils pas les ingrédients recherchés autant du côté de la villégiature que de la banlieue, encore aujourd'hui ${ }^{74}$ ? S'ils ont connu des altérations, subi des aménagements et des transpositions diverses, il n'empêche que ces éléments demeurent très présents. C'est une des principales raisons pour lesquelles les publicités, autant celles d'hier que d'aujourd'hui, se ressemblent autant. Ce qu'il importe alors de scruter, c'est tout le travail d'expérimentation et d'adaptation mené par ces divers groupes sociaux afin de saisir les objectifs qu'ils ont poursuivis, leurs rêves et leurs aspirations et tenter de mettre au jour les dynamiques entourant la mise à contribution de la nature et son exploitation à chaque époque.

74. Un article paru récemment atteste de la prégnance des aspirations tout comme des pratiques liées à la résidence secondaire: Bernard Cassen, «Au cœur de la Dordogne anglaise. Gentlemen et roturiers au pays de Cocagne», Le monde diplomatique, août 2004, 24. 